\title{
TAPSE/PASP use in heart failure: Implementing the evidence for simplicity
}

\author{
Marco Guazzi \\ University Cardiology Department, Policlinico San Donato, University of Milan, Italy
}

"Out of intense complexities intense simplicities emerge" Winston Churchill

The needle of balance and the limits between complexity and simplicity are generally quite clear but when things get too much hard and complicated simplicity emerges and compensates. This reminder by Winston Churchill appropriately applies to what clinicians have often to face and realize in their daily practice activities. The study of the right heart mechanics and its coupling with the pulmonary circulation in cardiopulmonary disorders is an example of this concept [1].

In cardiopulmonary disorders, when the right heart fails the prognosis becomes extremely poor [2]. Therefore, a close monitoring of the progressive changes in function over time becomes essential. The study of the right heart in terms of morphology and function is complex for a number of reasons: the peculiar anatomy and geometry which precludes a thorough definition in a two dimension; the predominant free-wall longitudinal motion with the overall contractile function dependent on the shared fibers with the left ventricle through the interventricular septum; the weak tolerance and compensatory response to pressure overload [3].

These reasons have been well defined as limitations for years, contributing to overlook some fundamental hemodynamic details [4]. Nowadays, the many hurdles in the study of the right ventricular (RV) geometry and, in part, function have been solved by the

Correspondence: Marco Guazzi, Professor of Cardiology, University of Milano School of Medicine, Head of Cardiology University Department, I.R.C.C.S. Policlinico San Donato, San Donato Milanese (MI), Italy.

Tel.-Fax: +39.02.52774966

E-mail: marco.guazzi@unimi.it

Commentary to: "Assessment of right ventricular-arterial coupling by echocardiography in patients with heart failure with reduced to midrange ejection fraction: impact on survival" by Rosa et al.

Received for publication: 8 May 2020.

Accepted for publication: 11 May 2020.

${ }^{\circ}$ Copyright: the Author(s), 2020

Licensee PAGEPress, Italy

Monaldi Archives for Chest Disease 2020; 90:1363

doi: 10.4081/monaldi.2020.1363

This article is distributed under the terms of the Creative Commons Attribution Noncommercial License (by-nc 4.0) which permits any noncommercial use, distribution, and reproduction in any medium, provided the original author(s) and source are credited. availability of advanced imaging techniques, although the pressure-volume analysis with Ees and Ea measures, obtained during evocative loading maneuvers, remains the gold standard for a precise measure of the RV to pulmonary circulation coupling.

Nonetheless, costs and limited availability for advanced imaging and the invasive nature of the pressure-volume curve may still limit the feasibility in the study of the RV systolic function and its coupling with the pulmonary circulation on a daily basis. For these reasons, the use of M-mode by tricuspid annular plane systolic excursion (TAPSE) or 2-D echo derived measures (right fractional area), tissue Doppler or speckle tracking analyses still represent the most common approaches for measuring the RV function [5]. These measures, although prognostic, may suffer from the loaddependency and may not define the coupling with the pulmonary circulation [1]. A simple method to circumvent these drawbacks recently proposed by our group is to normalize the TAPSE for the pulmonary arterial systolic pressure (PASP), which determines $\mathrm{RV}$-arterial coupling, as TAPSE is a surrogate of contractile function and PASP largely reflects afterload. The TAPSE/PASP ratio was originally tested in a group of heart failure (HF) patients with both preserved and reduced ejection fraction [6], which performed quite well at a prospective follow up in terms of cardiac-related mortality definition, emerging as an independent event predictor, stronger than other validated prognosticators, such as NYHA functional class, NT-pro-BNP levels, E/e' ratio. Most recent studies by ourselves and other groups have then validated TAPSE/PASP ratio against Ees and Ea [7,8] and its use has been extended out of HF to populations with PAH [9] and other pulmonary vascular diseases [10].

In the issue 2 of the Journal, Rosa et al. [11] report a large number analysis on TAPSE/PASP ratio prognostic prediction in $400 \mathrm{HF}$ with $<50 \%$ left ventricular ejection fraction patients. The cardiovascular death rate at 2 years follow-up was $34 \%$ and the TAPSE/PASP emerged as highly prognostic, confirming the favorable evidence on the use of this ratio as surrogate of RV to pulmonary circulation uncoupling in HF.

What is the novel information by this case series report? Substantially, these data reproduce the well-known clinical and prognostic definition of TAPSE/PASP, without providing any novelty in term of pathophysiology or specific subgroup analyses, except for the investigation of a population $>70$ years. Nonetheless, this remains a quite useful report because lends further credit on the clinical relevance of TAPSE/PASP use, widening the evidence to an additional HF case series.

Reproducibility of data was not tested, which seems an important limitation combined with the retrospective nature of this analysis. When the needle of the balance turns into simplicity, the increasing and consistent evidence across different laboratories strongly pushes for a widen use and implementation of TAPSE/PASP ratio as a simple "ready to go" measure of the right heart mechanics to be framed in the multifaced journey of our HF patients. 


\section{References}

1. Guazzi M and Naeije R. Pulmonary hypertension in heart failure: Pathophysiology, pathobiology, and emerging clinical perspectives. J Am Coll Cardiol 2017;69:1718-1734.

2. Guazzi M. The alarming association between right ventricular dysfunction and outcome: aetiology matters. Eur Heart J 2020;41:1283-1285.

3. Konstam MA, Kiernan MS, Bernstein D, et al. American Heart Association Council on Clinical C, Council on Cardiovascular Disease in the Y, Council on Cardiovascular S and Anesthesia. Evaluation and Management of Right-Sided Heart Failure: A Scientific Statement From the American Heart Association. Circulation 2018;137:e578-e622.

4. Amsallem M, Mercier O, Kobayashi Y, et al. Forgotten no more: A focused update on the right ventricle in cardiovascular disease. JACC Heart Fail 2018;6:891-903.

5. Rudski LG, Lai WW, Afilalo J, et al. Guidelines for the echocardiographic assessment of the right heart in adults: a report from the American Society of Echocardiography endorsed by the European Association of Echocardiography, a registered branch of the European Society of Cardiology, and the Canadian Society of Echocardiography. J Am Soc Echocardiogr 2010;23:685-713; quiz 786-8.

6. Guazzi M, Bandera F, Pelissero G, et al. Tricuspid annular plane systolic excursion and pulmonary arterial systolic pressure relationship in heart failure: an index of right ventricular contractile function and prognosis. Am J Physiol Heart Circ Physiol 2013;305:H1373-81.

7. Guazzi M, Dixon D, Labate V, et al. RV Contractile function and its coupling to pulmonary circulation in heart failure with preserved ejection fraction: Stratification of clinical phenotypes and outcomes. JACC Cardiovasc Imagin. 2017;10:12111221.

8. Tello K, Wan J, Dalmer A, et al. Validation of the tricuspid annular plane systolic excursion/systolic pulmonary artery pressure ratio for the assessment of right ventricular-arterial coupling in severe pulmonary hypertension. Circ Cardiovasc Imaging 2019;12:e009047.

9. Tello K, Axmann J, Ghofrani HA, et al. Relevance of the TAPSE/PASP ratio in pulmonary arterial hypertension. Int $\mathrm{J}$ Cardiol 2018;266:229-235.

10. Guo X, Lai J, Wang H, et al. Predictive value of non-invasive right ventricle to pulmonary circulation coupling in systemic lupus erythematosus patients with pulmonary arterial hypertension. Eur Heart J Cardiovasc Imaging 2019. doi: 10.1093/ehjci/jez311

11. Rosa GM ,D'Agostino A, Giovinazzo S, et al. Assessment of right ventricular-arterial coupling by echocardiography in patients with heart failure with reduced to mid-range ejection fraction: impact on survival. Monaldi Arch Chest Dis 2020;90:1269. doi: 10.4081/monaldi.2020.1269 